\title{
The Impact of Family Based Scheduling on Transfer Batches in a Job Shop Manufacturing Cell
}

Vijay R. Kannan

Information \& Decision Sciences Department, College of Business, James Madison University, Harrisonburg, VA 22807.

Steven B. Lyman

Department of Management, The Eli Broad Graduate School of Management, Michigan State University,

East Lansing, MI 48824-1121.

\begin{abstract}
Past research has shown that the performance of manufacturing cells can be improved if family oriented scheduling heuristics are used or if jobs are split into smaller transfer batches for processing and material handling purposes. The research has also shown that large amounts of lot splitting results in increased setup frequency due to the reduction in transfer batch size. This can offset any gains in performance. This study examines the combined effect of lot splitting in a manufacturing cell that utilizes family based scheduling heuristics. The results show that family based scheduling is an effective means of reducing the negative impact of lot splitting on flow time. Lot splitting however has little benefit for due date performance even when scheduling rules that reduce setup frequency are used.
\end{abstract}

Keywords: Cellular Manufacturing, Group Technology, Scheduling, Simulation 


\section{INTRODUCTION}

With demand patterns increasingly characterized by shorter product life cycles, greater variety and more frequent product revisions, a need exists for manufacturing organizations to reduce production lead times and to respond more quickly to changes in market demand. Not only does this make such organizations more competitive but it allows reductions to be made in work in process and finished goods inventories. Two approaches that have been used to achieve these objectives in shops using manufacturing cells are group scheduling and lot splitting.

The cellular manufacturing literature contains several examples of group scheduling rules that recognize similarities in setup requirements of jobs when making scheduling decisions. These rules typically schedule jobs using the same setup before changing the setup, thereby reducing setup frequency. Splitting jobs into smaller batches and allowing them to move independently has also been shown to reduce lead times. Lot splitting promotes a more continuous flow of materials and enables jobs to be processed simultaneously at different machines, lowering work in process and the occurrence of bottlenecks.

This research examines the effect of lot splitting when dynamic group scheduling rules are used in a job shop cell. An important tradeoff exists between transfer batch size and setup frequency in this environment. Small transfer batches improve material flows but reduce the amount of work sharing a setup. This results in more frequent setups but short delays encountered by batches awaiting a setup change. The use of group scheduling rules can help offset this increase in setup frequency, but this increases the length of delays faced by batches requiring a change in setup. When transfer batches are large, material flows are more lumpy and longer delays are faced by jobs requiring a setup change. Setup frequency is also lower. Though group scheduling rules can further reduce setup frequency, this compounds the problem of delays faced by batches requiring a change in setup. Two questions need to be addressed regarding the combined use of lot splitting and group scheduling in this environment: are group scheduling rules an effective means of overcoming the increased setup frequency attributable to lot splitting, and does lot splitting compromise the effectiveness of group scheduling? 


\section{PAST RESEARCH}

\subsection{LOT SPLITTING LITERATURE}

The benefits of lot splitting have been demonstrated by its use in the OPT production planning system (Fox, 1983). More recent research has focused on simulation studies of the impact of lot splitting in job and flow shops and manufacturing cells, and procedures for determining the size of transfer batches.

The repetitive lots procedure (Jacobs \& Bragg, 1988) for job shop scheduling permits jobs to move between machines as transfer batches that are a fraction of the job size. At each machine, transfer batches using the current setup are processed first before a setup change takes place. The procedure yielded significant improvements in performance compared to when lots were not split, even when transfer batches were a small fraction of the release batch size. Hancock (1991) considered a heuristic that allows a job to split at most once into two equal lots but not necessarily at the beginning of its routing as is typical of most lot splitting heuristics. This heuristic performed well over a range of conditions. Smunt et al. (1992) examined the performance of several lot splitting heuristics under different demand and shop conditions. They again showed that lot splitting yielded lower flow times. However, how lots were split was not significant. Of greater importance was the number of resulting transfer batches.

Karmarkar et al., (1985a) examined the impact of lot size in a manufacturing cell using both simulation and queuing theory. They showed that some reduction in lot size yielded improvements in lead time and work in process. However, beyond a certain size, further reductions resulted in increases in the number of setups and queue sizes, and a degradation in performance. Morris \& Tersine (1989) investigated the use of cell loading in a five cell shop. At any instant, a single job with a transfer batch size of one was processed within a cell. At low arrival rates, this yielded better performance than when jobs were not split and multiple jobs were processed simultaneously. However, as the arrival rate increased, overall utilization was low, resulting in poor performance. Sassani (1990) also showed that in a cellular shop, reducing transfer batch sizes yielded improvements in overall shop performance. However, the results showed that the performance yielded by individual cells was very sensitive to the processing characteristics of the jobs it processed.

A number of authors have attempted to optimize transfer batch size. Karmarkar et al., (1985b) described a procedure to determine the optimal lot size in a multi-stage production 
environment and incorporated this in an optimization algorithm. Moily (1986) developed optimal and heuristic lot splitting procedures for static multi-stage problems. Graves and Kostreva (1986) developed a model that optimizes transfer batch size based on cost for a two work station system in an MRP environment. Baker (1987), Trietsch (1987a, 1987b), and Baker and Pyke (1988) optimized transfer batch size based on makespan for a single job in a flow shop. Kropp and Smunt (1990) developed a model to optimize transfer batch size for a single job in a flow shop based on makespan and mean flow time. In addition, they considered the effect of setup time on optimal lot splitting, and identified computationally easy heuristics for the lot splitting problem.

\subsection{GROUP SCHEDULING LITERATURE}

Several simulation studies have examined the use of group scheduling heuristics in job shop cells. Vaithianathan \& McRoberts (1982) showed that a number of these heuristics yielded lower flow times and number of setups per job than shortest processing time (SPT) dispatching. However, the due date performance of these heuristics was very poor. Mosier (1983) and Mosier et al., (1984) showed that a rule that selects a family for processing based on greatest work content of jobs in the current queue yielded the greatest improvement over traditional dispatching rules. This was closely followed by a rule that evaluates the economic benefit of changing setups at the completion of each job. They obtained significant flow time improvements by using family based scheduling but more limited improvements in due date performance.

Flynn (1987) applied the repetitive lots procedure in a five cell shop and showed that its use yielded significant improvements in performance over first come first served (FCFS) dispatching. Mahmoodi et al., (1990) showed that rules that select the next family based on the due date of the most imminent job, and on minimizing all future setups, performed well for most performance measures. The relative performance of the family based heuristics considered depended on other shop conditions, for example load and the ratio of setup time to processing time. In an earlier work (1988) they showed that family scheduling heuristics based on either the due date of the most imminent job or the earliest job to arrive at the queue, always yielded superior flow time and tardiness performance compared to scheduling using the corresponding dispatching rule alone. 
Mahmoodi \& Dooley (1991) compared exhaustive heuristics that process all jobs that require the current setup before a setup change occurs, to non-exhaustive rules where a setup change can occur even though jobs remain in the queue that require the current setup. Their results showed that exhaustive rules typically yielded the best performance, but that for due date performance, non-exhaustive rules can perform at a comparable level. Ruben et al., (1993) showed that a rule that selects the next family based on minimum setup time and dispatches jobs in this family using the SPT dispatching rule, outperformed exhaustive rules used previously by Mosier and Mahmoodi with respect to mean flow time and proportion tardy. They also showed that the gains from the use of group scheduling rules were greater when setup time was high and utilization was high. They confirmed that group scheduling has a greater impact on flow time performance than tardiness performance. Kannan \& Lyman (1992) compared family scheduling heuristics that select a family based on the characteristics of a single job to those that use a corresponding composite measure of the family. They showed that family selection based on job characteristics is as effective as selection based on family characteristics. The objective of the current research is to more extensively examine the combined use of lot splitting and group scheduling in a manufacturing cell. Past research, has demonstrated the benefits associated with lot splitting but has suggested that the corresponding increase in setups may be a limiting factor. The use of scheduling heuristics that attempt to minimize setup frequency represents one possible way to overcome this problem. Existing group scheduling research has paid only limited attention to the effects of lot size. This study examines different lot splitting strategies in conjunction with group scheduling heuristics that have been shown in the past to perform well.

\section{SIMULATION ENVIRONMENT}

The shop modelled here is the same as that used by Mahmoodi et al., (1990). This is a five work station job shop cell with one machine at each work station (Figure 1). Incoming jobs are initially processed at either machine 1 or 2 , pass through machine 3 , and exit from either machine 4 or 5 . This yields sixteen distinct routings. No more than one operation takes place at a given machine. The use of a bottleneck machine is consistent with prior studies of job shops that suggest that machine utilizations are not balanced (Fry et al., 1988).

As in the Mahmoodi articles, three part families are processed within the cell. These are distinguished by different setup requirements. The three families and sixteen routings yield a 
total of forty eight parts. Incoming jobs have an equal probability of being for each of the part types. Jobs arrive according to a Poisson process (Law \& Kelton, 1982). Inter-arrival times are thus exponentially distributed. Job size is one hundred units (Kropp \& Smunt, 1990) Processing times are distributed normally with a mean of one hour and a standard deviation of .25 hours (Mahmoodi et al., 1992). Job due dates are established using the total work content rule (Conway et al., 1967) with an allowance of 2.75 (Weeks \& Fryer, 1977).

\section{EXPERIMENTAL DESIGN}

Four experimental factors are considered in this study. In addition to examining the effect of different family scheduling rules and transfer batch sizes, two factors that have been shown in the past to affect the performance of manufacturing cells are included. These are setup times and dispatching rules. Three family selection rules that have been used in past group scheduling research are examined. The family selected is that with the highest total work content. This rule was suggested by Mosier et al., (1984), and was shown to be the best performing rule in their study. The family corresponding to the first transfer batch to arrive at the queue is selected. This rule was first used by Flynn (1987) and is the basis of the repetitive lots procedure. The family selected is that corresponding to the transfer batch with the lowest job slack (Kannan \& Lyman, 1992).

A fourth level of this factor, NOFAM, is included which corresponds to non-family based scheduling. Scheduling decisions are made based on the dispatching rule alone. This is included in order to demonstrate whether any benefits or limitations from the use of lot splitting could have been obtained without using family based scheduling. Transfer batches requiring the current setup are dispatched using one of three rules:

FCFS: First Come First Served. This is a commonly used rule in practice due to its ease of use (Conway et al., 1967).

SPT: Shortest Imminent Processing Time. In past job shop and group scheduling research, this rule has been shown to perform well particularly with respect to mean flow time (Conway et al., 1967, Mosier et al., 1984).

MINSLK: Minimum Job Slack. This rule has been shown in the past to perform well in both job and cellular shops, particularly for due date measures (Conway et al., 1967, Mosier et al., 1984). 
In order to investigate the impact of lot splitting, three transfer batch sizes are considered Large Transfer batch size is 100 . This is equivalent to not splitting release batches Medium Transfer batch size is 50. Release batches are split into two transfer batches of the same size. Small Transfer batch size is 25 . Release batches are split into four transfer batches of the same size.

All lot splitting occurs at the start of a job's routing. Since the focus of this research is on the underlying impact of lot splitting when group scheduling is used and not on lot sizes themselves, only transfer batches of equal size are considered. This is consistent with the finding of Smunt et al., (1992) that how lots are split is of less importance to shop performance than the number of transfer batches. However, evidence suggests that benefits may be derived from the use of transfer batches of unequal size (e.g., Fox 1983). Mean job inter-arrival times are established to yield an average utilization of $90 \%$ for the bottleneck machine when non-family based FCFS dispatching is used with small transfer batches.

Two values of setup time are investigated. Only major (between family) setups are considered. Within a family, changes in setup are assumed to be small relative to major setups and thus their effect insignificant. This is consistent with previous research (e.g., Mahmoodi et al., 1990). Setup times are distributed normally with a mean of either 0.33 or 0.66 hours and a coefficient of variation of 0.25 . These yield setup time/run time (S/R) ratios of 0.33 and 0.66 (Mahmoodi et al., 1992).

A full factorial design was used with seventy two $(4 * 3 * 3 * 2)$ treatments. Data was collected for four performance measures: the mean and standard deviation of flow time and tardiness. In order to meet assumptions of batch independence and normality, data was collected for each treatment from eleven batches of 1600 jobs. The first batch was discarded in each case to eliminate the effects of initialization bias. These run characteristics were obtained from pilot runs. Common random numbers were used to reduce variance. Independence between treatments was obtained by not synchronizing one random number stream, and using this random number stream as a blocking factor (Mihram, 1974).

The simulation was modelled using the SIMAN simulation language (Pegden, 1987) and user written FORTRAN subroutines. All statistical analysis was conducted using the SYSTAT statistical software package at a significance level of $=0.05$. 


\section{RESULTS}

Treatment means are reported in Tables 1-4. Analysis of residuals indicated that assumptions underlying the use of analysis of variance (ANOVA) were met. As described previously, the unsynchronized random number stream was used as a blocking factor to ensure the independence of treatments. ANOVA results (Table $5, *$ denotes significance at the $=0.05$ level) show that for all performance measures, all main effects are significant except those due to the setup time ratio. This is significant only for mean flow time. As expected, it shows performance to be better when setup time is low. In addition, all two way interactions between combinations of family selection rule, transfer batch size and dispatching rule, and the three way interaction between these factors are significant. To identify the impact of these effects, Tukey multiple comparisons of treatment means were carried out by dispatching rule for all combinations of family selection rule and transfer batch size (Figure 2). This allows the trade-off between transfer batch size and the use of setup reducing scheduling mechanisms to be seen.

For all performance measures, FCFS dispatching yields similar performance for all family selection rule $x$ transfer batch size combinations when transfer batch size is medium or large or when transfer batch size is small and family based scheduling is not used (Figures 3a-d). Performance is significantly better under these conditions than when transfer batch size is small and either of the three rules that recognize families is used. These three treatments yield similar, very poor performance. Recognizing families has a significant impact only when transfer batch size is small, and then it is an adverse impact. For other transfer batch sizes, family based scheduling again yields poorer performance but this is not significantly poorer than when nonfamily based scheduling is used.

When SPT dispatching is used, mean flow time is lowest when transfer batch size is small and family based scheduling is used (Figure 4a). This is followed by the performance obtained when transfer batches are of medium size and family based scheduling is used or when small transfer batches are used with non-family based scheduling. Performance is poorest whenever large transfer batches are used. There are no significant differences in performance between family selection rule $\mathrm{x}$ transfer batch size combinations for the standard deviation of flow time (Figure 4b). However, small transfer batches generally yield marginally higher values and family based scheduling marginally lower values. For both tardiness measures, performance is best whenever transfer batches are large (Figures $4 \mathrm{c}, \mathrm{d}$ ). Small transfer batches yield the 
poorest performance. Though the use of family based scheduling consistently yields lower values for both measures of tardiness performance, there are few significant differences compared to non-family based scheduling. The only significant differences are that for small transfer batches, both measures are lower when family based scheduling is used, and for medium sized transfer batches, the same applies for the standard deviation of tardiness.

When MINSLK dispatching is used, mean flow times are again lowest when transfer batches are small and highest when transfer batches are large (Figure 5a). However, family based scheduling, though yielding lower values, is not significantly better. Results for the standard deviation of flow time indicate that it is lowest whenever transfer batches are of small or medium size and family based scheduling is used (Figure $5 \mathrm{~b}$ ). Poor performance is obtained whenever transfer batches are large. For both tardiness measures, performance is best when transfer batches are large and poorest when they are small (Figures 5c,d). As with SPT dispatching, there are few significant differences due to the use of family based scheduling. Though family based scheduling generally yields lower values, the only significant difference is that when small transfer batches are used, the standard deviation is lower when family based scheduling is used.

\section{DISCUSSION}

The results confirm those found in previous studies as well as providing new insights into the relationship between batch size and scheduling mechanisms in this environment. It is no surprise that flow time is lower when setup time is low (Mahmoodi et al., 1990). Similarly, the relatively good performance of the SPT and MINSLK dispatching rules is not a new observation (Mahmoodi et al., 1990).

The particularly poor performance of the FCFS dispatching rule when transfer batch size is small and family based scheduling is used is of interest. Under these conditions, the relative inefficiency of the rule does not enable it to consistently clear queues. This appears to result in high variance in queue lengths. Transfer batches that arrive at machines when queues are long or contain many batches from a different family that is currently using the machine, face lengthy delays. Conversely, batches that arrive when queue lengths are short or when batches from their family are being processed, incur shorter delays. Since only one transfer batch of a job need to be delayed to delay completion of the job, high flow time variance results. This is more likely to occur when there are more transfer batches that can be delayed, i.e., when transfer batches are 
small. When family based scheduling is not used, jobs do not have to wait for all batches using the current setup to be processed, thus the delay while awaiting a setup change is shorter. However, batches encounter more frequent setup delays. These appear to be shorter in length, resulting in relatively lower flow time variance.

The observation that the adverse impact of setup sharing is not found when transfer batches are of medium or large size appears to suggest that transfer batches accumulate more when transfer batches are small even though batch processing times are proportional to batch size. This suggests a paradox. Improving the flow of transfer batches but allowing them to combine where this is appropriate is one of the goals of lot splitting. However when lots are small, allowing them to combine to obtain setup efficiencies is what causes poorer performance.

The results for the SPT and MINSLK dispatching rules more clearly show the potential benefits of group scheduling in this environment. As expected, flow time performance is better when transfer batches are small. Jobs are processed more efficiently since they can move between machines with shorter delays while awaiting other units of the batch to be processed. Processing of a job can also occur concurrently at different machines. The results presented here show that though differences are not always significant, the use of family scheduling always lowers flow times regardless of transfer batch size. Transfer batches are not faced with a setup at each machine as they are when single stage dispatching rules are used. This is what in the past has compromised the benefits of lot splitting. By allowing batches to be effectively combined into larger batches depending on prevailing conditions, the lower setup frequency normally associated with larger batches is obtained. This occurs without the lumpiness of material flows obtained when jobs themselves move as single large batches. Family based scheduling allows the shop to determine when large batches are appropriate as opposed to having this constraint imposed on it. In doing so, family based scheduling bridges the gap between using the planning system and dispatching rules to reduce problems associated with shop floor control.

The benefits of lot splitting on due date performance are less apparent. For both the SPT and MINSLK dispatching rules, tardiness performance degrades as transfer batch size is reduced. Decreasing the transfer batch size increases the number of transfer batches comprising a job that could potentially delay its completion. The use of family based scheduling has less impact on tardiness performance. This is not unexpected given the results of earlier studies of group scheduling (e.g., Ruben et al., 1993). Though family based scheduling consistently yields lower 
values for both measures of tardiness, differences compared to non-family based scheduling are generally small in magnitude. Only when transfer batch size is reduced are any significant benefits obtained. This again suggests that the benefits of family scheduling increase as the congestion associated with reducing transfer batch size increases.

\section{CONCLUSIONS}

Group scheduling can be an effective way to reduce the negative impact on flow time performance of lot splitting in job shop cells. If control mechanisms within the shop are able to prevent the build up of queues, reductions in batch size and thus the inherent lumpiness of batch production promotes more efficient material handling and processing. Further, when scheduling rules take advantage of processing similarities between successive batches, the material handling benefits of small batches are obtained without sacrificing the setup benefits of large batches. Equally significant is that the splitting of batches does not appear to constrain the efficiencies obtained by recognizing family affiliations. Though small batch sizes compromise due date performance to an extent that family scheduling cannot compensate for, the use of family based scheduling does not in general cause declines in performance for either flow time or due date measures. Current manufacturing trends continue to emphasize small batch sizes, work in process inventories and lead times, and a move towards a just-in-time (JIT) orientation. The combined use of lot splitting and group scheduling appears to be an effective way for small batch manufacturers to achieve some of these objectives of JIT systems without significantly changing the nature of the production system. However, the improvements in performance obtained in this study also demonstrate the need to place greater attention on the planning system itself rather than using family scheduling rules as a surrogate.

\section{REFERENCES}

Baker, K.R., 1987, Lot Streaming to Reduce Cycle Time in a Flow Shop, Working Paper, Amos Tuck School, Dartmouth College .

Baker, K.R., Pyke, D.F., 1988, Algorithms for the Lot Streaming Problem, Working Paper, Amos Tuck School, Dartmouth College.

Conway, R.W., Maxwell, W.L., Miller, L.W., 1967, Theory of Scheduling, Addison Wesley. 
Flynn, B.B., 1987, Repetitive Lots : The Use of a Sequence Dependent Set-Up Time Scheduling. Procedure in Group Technology and Traditional Shops, Journal of Operations Management, 7 (1 \& 2), 203-216.

Fox, R.E., 1983, OPT - An Answer for America - Part IV, Inventories and Production Magazine , 3 (2).

Fry, T.D., Philipoom, P.R., Markland, R.E., 1988, Dispatching in a Multi-Stage Job Shop Where Machine Capacities are Unbalanced, International Journal of Production Research , 26, 1193-1223.

Graves, S.C., Kostreva, M.M., 1986, Overlapping Operations in Materials Requirements Planning, Journal of Operations Management , 3 (2), 283-294.

Hancock, T.M., 1991, Effects of Lot Splitting Under Various Routing Strategies, International Journal of Operations and Production Management, 11, 68-74.

Jacobs, F.R., Bragg, D.J., 1988, Repetitive Lots : Flow Time Reductions Through Sequencing and Dynamic Batch Sizing, Decision Sciences , 19 (2), 284-291.

Kannan, V.R., Lyman, S.B., 1992, A Comparison of Family and Job Based Priority Schemes in Group Scheduling, Proceedings of the National Decision Sciences Meeting, San Francisco, CA , 1454-1456.

Karmarkar, U.S., Kekre, S., Kekre, S., Freeman, S., 1985a, Lot Sizing and Lead Time Performance in a Manufacturing Cell, Interfaces , 15 (2), 1-9.

Karmarkar, U.S., Kekre, S., Kekre, S., 1985b, Lot Sizing in Multi-Item Multi-Machine Job Shops, IIE Transactions , 17 (3), 290-297.

Kropp, D.H., Smunt, T.L., 1990, Optimal and Heuristic Lot Splitting in a Flow Shop, Decision Sciences , 691-709.

Law, A.M., Kelton, W.D., 1982, Simulation Modeling and Analysis, McGraw Hill .

Mahmoodi, F., Dooley, K.J., Starr, P.J., 1988, A Comparison of Dynamic Scheduling Heuristics for Cellular Manufacturing, Proceedings of the National Decision Sciences Meeting, Las Vegas, NV, 893-895.

Mahmoodi, F., Dooley, K.J., Starr, P.J., 1990, An Investigation of Dynamic Group Scheduling Heuristics, International Journal of Production Research , 28 (9), 1695-1711. 
Mahmoodi, F., Dooley, K.J., 1991, A Comparison of Exhaustive and Non-Exhaustive Group Scheduling Heuristics in a Manufacturing Cell, International Journal of Production Research , 29 (9), 1923-1969.

Mahmoodi, F., Tierney, L.J., Mosier, C.T., 1992, Dynamic Group Scheduling Heuristics in a Flow-Through Cell Environment, Decision Sciences , 23 (1), 61-85.

Mihram, A., 1974, Blocking in Simular Experimental Designs, Journal of Statistical Computing and Simulation , 3, 29-32.

Moily, J.P., 1986, Optimal and Heuristic Procedures for Component Lot Splitting in Multi-Stage Manufacturing Systems, Management Science, 32 (1), 113-125.

Morris, J.S., Tersine, R.J., 1989, A Comparison of Cell Loading Practices in Group Technology, Journal of Manufacturing Operations Management , 2, 299-313.

Mosier, C.T., 1983, The Job Shop Using Group Technology : A Simulation Investigation, Ph.D Dissertation, University of North Carolina .

Mosier, C.T., Elvers, D.A., Kelly, D., 1984, Analysis of Group Technology Scheduling Heuristics, International Journal of Production Research , 22 (5), 857-875.

Pegden, C.D., 1987, Introduction to SIMAN, Systems Modeling Corporation .

Ruben, R.A., Mosier, C.T., Mahmoodi, F., 1993, A Comprehensive Analysis of Group Scheduling Heuristics in a Job Shop Cell, International Journal of Production Research , 31 (6), 1343-1370.

Sassani, F., 1990, A Simulation Study on Performance Improvement of Group Technology Cells, International Journal of Production Research , 28 (2), 293-300.

Smunt, T.L., Kropp, D.H., Buss, A.H., 1992, Lot Splitting in Stochastic Flow Shop and Job Shop Environments, Working Paper, John M. Olin School of Business, Washington University Trietsch, D., 1987a, An Efficient Transfer Lot Sizing Procedure for Batch Processing on Several Machines, Working Paper, Naval Postgraduate School .

Trietsch, D., 1987b, Optimal Transfer Lots for Batch Processing: A Basic Case and Extensions, Working Paper, Naval Postgraduate School .

Vaithianathan, R., McRoberts, K.L., 1982, On Scheduling in a GT Environment, Journal of Manufacturing Systems , 1, 149-155.

Weeks, J.K., Fryer, J.S., 1977, A Methodology for Assigning Minimum Cost Due Dates, Management Science, 23 (8), 872-881. 